\title{
The Effect of Increased Microporosity on Bone Formation within Silicate-Substituted Scaffolds in an Ovine Posterolateral Spinal Fusion Model.
}

\author{
Melanie J Coathup, BSc, $\mathrm{PhD}^{1}$, Gordon W Blunn BSc, $\mathrm{PhD}^{1}$, \\ Charlie Campion BMedSci ${ }^{2,3}$, Chih-Yuan Ho BSc, $\mathrm{PhD}^{1}$ \\ and Karin A Hing BSc, $\mathrm{PhD}^{2}$.
}

\section{Corresponding author and author for reprint requests:}

Dr Melanie Coathup

John Scales Centre for Biomedical Engineering, Institute of Orthopaedics and Musculoskeletal Science, Division of Surgery and Interventional Science, University College London, The Royal National Orthopaedic Hospital, Brockley Hill, Stanmore Middlesex. HA7 4LP. United Kingdom. Tel: +44 208 954 5264, Fax: +44 208420 7392, e-mail: m.coathup@ucl.ac.uk.

\begin{abstract}
This study compared the bone forming capacity of the same formulation of silicate-substituted bone graft substitute materials with different microporosity in an instrumented posterolateral spinal fusion ovine model. Materials with a strut porosity of (i) $22.5 \%(\mathrm{SiCaP})$ or (ii) $36.0 \%(\mathrm{SiCaP}(+))$ were packed along either side of the spine. Bone apposition rates, \% new bone formation, $\%$ bone-scaffold contact and $\%$ graft resorption were quantified at 8, 12 and 24 weeks post surgery. Computed Tomography (CT) was used to grade the formation of fusion bridges between vertebrae. Results showed no significant difference in bone apposition rates, $\%$ new bone formation and $\%$ bone-implant contact when the two materials were compared. However, at 8 weeks, a significantly higher CT score was obtained in the $\mathrm{SiCaP}(+)$ group $(0.83 \pm 0.17)$ when compared with the $\mathrm{SiCaP}$ group $(0.17 \pm 0.17 ; \mathrm{p}=0.027)$. Significantly less scaffold remained in the $\mathrm{SiCaP}(+)$ group at 12 weeks $(\mathrm{p}=0.018)$. Both $\mathrm{SiCaP}$ and
\end{abstract}




\section{Effect of Microporosity on Bone Formation}

$\mathrm{SiCaP}(+)$ formulations augmented bone formation. Increasing the strut porosity did not significantly increase bone formation however, at 8 weeks it promoted the formation of more highly mineralised bone resulting in a significantly higher CT score, suggesting the bone tissue formed was more mature.

Keywords: Bone regeneration; posterolateral spinal fusion; osteoconduction; strut porosity; calcium phosphate; sheep model; bone substitute materials.

\section{Introduction}

The most common application of bone graft is in posterolateral spinal fusion surgery, where the graft is used to create a bony bridge between the vertebrae that connects and stabilises the affected segments. The procedure is believed to be one of the most challenging indications for bone graft due to the large distance that needs to be bridged, limited contact surface available and the unfavourable biomechanical environment due to the lack of compression forces [1]. Presently autograft harvested from the iliac crest is considered the 'gold standard' for regenerating bone due to its biological performance in terms of osteoinductivity [2 - 4] and osteoconductivity [4-6]. However, the amount of iliac crest bone available is limited and its harvesting is associated with increased operative and recovery times and significant donor site morbidity $[7,8]$.

Calcium phosphate based ceramic bone graft substitute materials represent an alternative to autologous bone and have been successfully used in the repair of bony defects caused by trauma, malignancy and infection [7, $9-11]$. Several studies have shown that their bioactivity can be significantly augmented through chemical substitution of phosphate ions with silicate ions [12 - 14]. In a recent study by Wheeler et al. [15], the use of silicate-substituted calcium phosphate ( $\mathrm{SiCaP}$ ) graft in an instrumented posterolateral fusion model was reported to be biomechanically, radiographically and histologically equivalent to autograft, generating a solid, bony inter-transverse process fusion in an ovine model. In addition, two clinical studies that investigated the effect $\mathrm{SiCaP}$ in patients following spinal fusion 


\section{Effect of Microporosity on Bone Formation}

surgery, reported $90 \%$ [8] and 100\% [16] levels of bony fusion and concluded it to be an effective bone graft substitute that eliminated the need for autogenous iliac crest bone graft. Studies have since questioned whether the efficacy of $\mathrm{SiCaP}$ is due to the presence of silicon or the resulting microstructure $[17,18]$. A number of studies have reported the significant effect of silicate ions on augmenting bone formation [12 - 14] whilst other reports have shown that increasing the strut (micro)porosity of porous SiCaP significantly augments the repair, regeneration and bone-implant contact in both ectopic and orthotopic sites [19-22]. In this study, we asked the question whether a SiCaP scaffold with increased strut porosity would further enhance the regenerative bony capacity and increase bone bridging in an ovine posterolateral spinal fusion model. Our hypothesis was that silicate-substituted scaffolds with increased strut porosity would significantly increase bone formation and enhance fusion.

\section{Materials and Methods}

The two SiCaP scaffolds investigated (1) SiCaP and (2) $\mathrm{SiCaP}(+)$ (supplied by ApaTech Ltd, Herts, UK in the form of irregularly shaped $1-2 \mathrm{~mm}$ sized granules), had the same total macroporosity of $80-$ $82.5 \%$ with an average macropore diameter of $300 \mu \mathrm{m}$, and differed only in the level of strut porosity (micropores within the struts were $<50 \mu \mathrm{m}$ in diameter and had a typical diameter of 1 to $10 \mu \mathrm{m}$ ). Scaffolds in experimental Group $1(\mathrm{SiCaP})$ were manufactured with a strut porosity of $22.5 \pm 2.5 \%$ (mean with standard deviation of mean) and in Group $2(\mathrm{SiCaP}(+))$ materials had a strut porosity of $36.0 \pm 2.5 \%$. Both materials were composed of phase pure hydroxyapatite substituted with $2.6 \mathrm{wt} \%$ silicate (equivalent to $0.8 \mathrm{wt} \% \mathrm{Si}$ ).

Porous ceramic foams with varied strut porosity were manufactured via a foaming route [23] and the phase purity confirmed using X-ray diffraction (XRD) as previously described [23]. The calcium, phosphorus, and silicon contents were determined by X-ray fluorescence (XRF) [23] and total porosity was confirmed according to a water immersion densitometry method [24]. The strut-porosity was 
confirmed by mercury intrusion porosimetry in combination with helium pyconometry as detailed in our previous study [19].

Thirty-six bilateral, instrumented, posterolateral spinal fusion procedures were performed on 36 skeletally mature female commercially cross-bred sheep, $2-5$ years of age and weighing between $65-85 \mathrm{~kg}$. All procedures were carried out following Ethics approval granted by the Royal Veterinary College and in compliance with U.K Home Office regulations as stated in the Animal Scientific Procedures Act (1986). Animals were euthanised at 8, 12 or 24 weeks post surgery. A total of six animals implanted with each bone graft type were investigated in each group at each of the three time points.

\section{Surgery}

Animals were placed in dorsal recumbence and longitudinal incisions measuring approximately $6-8 \mathrm{~cm}$ were made over the spinous processes from L3 to L5. Through this skin incision, the subcutaneous muscle firstly to the right lateral side of the spinal column was divided by blunt dissection to expose the transverse processes. The transverse processes of L4 and L5 and the adjacent spine, laminae, posterolateral aspect of the pars and facet joints were cleared of soft tissue and the facet joint excised. Decortication of the dorsal aspect of each transverse process and lateral spine was carried out using a burr and pedicle screws inserted. An interconnected rod was applied and $8 \mathrm{cc}$ of bone graft material packed between the two transverse processes. The graft which bridged the two transverse processes, was placed onto the decorticated surfaces, around the dorsal facets, around the pedicle screws and inferior to the metallic rod implants (Nuvasive, San Diego, USA). The inter-granular distance was not measured and assumed to be the same in all cases as the packing technique was similar. The wound was then carefully closed in layers. Prior to closure of the skin, the procedure was repeated on the contralateral side. The graft material for one animal inserted onto the right and left hand sides of the spine was the same and surgeons were not blinded to the graft-type implanted. Following surgery, animals were administered with routine prophylactic antibiotics and analgesia and were allowed to mobilise as tolerated. Implants 


\section{Effect of Microporosity on Bone Formation}

remained in vivo for 8,12 or 24 weeks. These time-points were chosen as they are considered clinically relevant time-points. Fusion associated with bone formation in spinal fusion surgery has been shown to occur at 24 weeks and the 8 and 12 week time-points investigated in this study allowed us to assess the early and mid-bony response. All analyses were carried out blind to implant type.

\section{Bone Apposition Rates}

When administered in vivo, fluorochromes that absorb to bone mineral can be used as markers for quantifying the active mineralising surfaces of bone. When two or more markers with different emission spectra are administered at known time points, bone apposition rates ( $\mu \mathrm{m} /$ day) can be quantified by measuring the distance between markers when viewed under UV light. In this study, oxytetracycline (30 $\mathrm{mg} / \mathrm{kg}$ ) and Alizarin Red (30 mg/kg) were intravenously administered at 5 and 7 weeks in the 8 -week group, at 9 and 11 weeks in the 12-week study group and at 20 and 22 weeks in the 24-week group.

Following euthanasia, the spinal column between L3 - L6 was retrieved en bloc with surrounding muscle. Samples were fixed and preserved using $4 \%$ paraformaldehyde for 7 days and were then processed for undecalcified histology. After dehydration in a graded alcohol series and defatting using chloroform solution, the samples were embedded in polyhydroxy-aromatic acrylic resin (LR White ${ }^{\mathrm{TM}}$ from The London Resin Company, Reading, United Kingdom). The specimens were cut transversely through the affected lumbar region in the dorsal/ventral plane and thin sections $(\sim 60 \mu \mathrm{m})$ were prepared using an EXAKT grinding system (EXAKT Corp, Norderstedt, Germany). Longitudinal thin sections were also prepared through the cranial/caudal plane in each of the animals. Measurements were taken from 7 regions of interest within the graft material using fluorescence microscopy. Image analysis techniques (Axiovision 4.5; Carl Zeiss, Jena, Germany) were used to quantify the measurable differences between the two bone markers. 


\section{Histological Evaluation}

Following measurements for bone apposition rates sections were subsequently stained with Toluidine Blue and Paragon (stains soft tissue and bone, respectively). The sections were examined histologically using light microscopy (Figure 1). 'Bone formation within the bone graft substitute material was measured from a transverse section taken mid-way between the two transverse processes. Five images spanning the thin section were captured using a $5 \times$ objective lens and analyzed with use of image analysis software (AxioVision 4.5; Carl Zeiss, Jena, Germany). The line intersection method $[14,19,20]$ was used to quantify the percentage of bone within the graft material, and of implant material in the image. A $10 \times 12-\mathrm{mm}$ grid of lines was placed over the image. The type of material present at each of the 225 intersection points of the grid lines was determined. Bone in direct contact with the implant surface (bone-implant contact) was determined at the points where the lines intersected with the $\mathrm{SiCaP}$ or $\mathrm{SiCaP}(+)$ biomaterial. The percentage of tissue, material and bone-implant contact was then calculated as a mean from a set of five images of each implant and statistical comparisons were performed.'Bone was not quantified in pores $<50 \mu \mathrm{m}$. In order to investigate whether bone formation had resulted in fusion of the spine, sections orientated longitudinally (cranial-caudal plane) between the vertebrae and the spinal rod were also made. As reported above, the spinal fusion mass was sectioned transversely (dorsal-ventral plane) and in order to obtain longitudinal histological images, three sections ( $\mathrm{A}, \mathrm{B}$ and $\mathrm{C}$ ) equivalent to a single longitudinal section were constructed.

\section{Computed Tomography}

Computed tomography (CT) was carried out on the 12 animals in the 24-week group only, at 8, 16 and 24 weeks post surgery. Multi-planar 3-Dimensional image reconstructions (in the coronal and sagittal plane) of axial CT images were carried out using QMA (Medical Metrics, Inc, Houston, Texas USA). The CT imaging protocol consisted of $0.6 \mathrm{~mm}$ thick and $0.3 \mathrm{~mm}$ overlapping axial slices with levels adjusted to 
optimise trabecular bone detail. An independent veterinary radiologist performed blinded CT assessments as follows:

\section{(i) Bridging Bone (Fusion)}

Evidence of bridging bone in CT images across the implanted posterior elements was semi-quantitatively graded in accordance with the following scoring system:

0. None - no evidence of callus formation associated with dorsal articular facets (articular surfaces well defined).

1. Minimal Callus Formation - Some callus formation is present however the callus is poorly defined (amorphous) and not consolidated between the facets (articular surfaces still well defined).

2. Partial Callus Formation - Callus formation is present and the callus appears better defined and is beginning to consolidate across the facets (articular surfaces lack some definition).

3. Fusion - Callus formation is present and it is well defined, consolidated and bridging between the facets (articular surfaces poorly defined).

\section{(ii) Adjacent Level Degeneration}

Evidence of adjacent level degeneration graded in accordance with the following definitions:

0 Absent - No evidence of disc mineralisation, osteophyte formation, endplate sclerosis and/or disc height loss at the level(s) adjacent to the treated level suggestive of degeneration.

1 Present - Presence of disc mineralisation, osteophyte formation, endplate sclerosis and/or disc height loss at the level(s) adjacent to the treated level suggestive of degeneration.

(iii) Implant Loosening or Infection 
Evidence of loosening of the implant or gross signs of infection at the treated level:

0. Absent - No Evidence of implant loosening or infection at the treated level.

1. Present - Presence of implant loosening or infection at the treated level

\section{Statistics}

The Mann-Whitney U test was used for statistical analysis between experimental groups where P values < 0.05 were considered significant. The Kruskal-Wallis test with post-hoc Mann-Whitney U tests were used to compare data at different time points and within one experimental group where $\mathrm{P}$ values $<0.05$ were considered significant (version 10.1; SPSS, Chicago, Illinois). Mean values are presented with standard error of mean.

\section{RESULTS}

\section{Bone Apposition Rates}

No significant difference in bone apposition rates within the bone graft were seen when the two experimental groups were compared at 8,12 and 24 weeks. Over a 24 week period, bone apposition rates decreased over time with lowest rates measured in both groups at the 24-week time-point (mean SiCaP, $1.33 \pm 0.23 \mu \mathrm{m} /$ day, mean $\mathrm{SiCaP}(+), 1.76 \pm 0.32 \mu \mathrm{m} /$ day) (Figure 2). In the $\mathrm{SiCaP}$ group, a significantly higher bone apposition rate was measured at 8 weeks $(2.93 \pm 0.33 \mu \mathrm{m} /$ day $)$ when compared with the 24 week time-point $(\mathrm{p}=0.010)$. No other significant differences were found.

\section{New Bone Formation}

Similar amounts of new bone formation were measured within the $\mathrm{SiCaP}$ and $\mathrm{SiCaP}(+)$ scaffolds and results showed no significant differences when the 8, 12 and 24-week time points were compared. Histological analysis revealed that new bone formation increased over time in both groups. In the SiCaP 


\section{Effect of Microporosity on Bone Formation}

group, significantly increased levels of bone formation was measured within the graft material at 12 weeks (mean, $46.28 \% \pm 0.68 \%)$ when compared with 8 week data (mean, $32.88 \% \pm 0.79 \%)(p=0.006)$. At 24 weeks significantly increased bone was measured (mean, 54.14\% $\pm 3.96 \%$ ) when compared with bone present 8 weeks post surgery $(\mathrm{p}=0.004)$. No significant difference was found when 12 and 24week data was compared. In the $\mathrm{SiCaP}(+)$ group, a significantly increased amount of bone was measured in the 24 week group (mean, $58.07 \% \pm 3.31 \%$ ) when compared with both the 8 week (mean, $32.75 \% \pm 2.39 \%)$ and 12 week groups (mean, $40.34 \% \pm 3.56 \%)(\mathrm{p}=0.003$ and $\mathrm{p}=0.016$ respectively). No significant difference was measured when the 8 and 12-week groups were compared.

\section{Bone-Graft Substitute Contact}

Results showed similar amounts of $\%$ bone-implant contact in the two experimental groups. No significant differences were found when the two groups were compared at any of the three time-points investigated in this study. Longitudinal analysis showed a trend where bone-implant contact increased over time in both groups. In $\mathrm{SiCaP}$ samples, significantly increased bone-implant contact was measured at 24 weeks (mean, $84.63 \% \pm 1.56 \%$ ) when compared with both the 8 -week group (mean, $60.76 \% \pm$ $1.73 \%)$ and 12 week group $(75.87 \% \pm 1.67 \%)(p=0.004$ and $\mathrm{p}=0.006$ respectively $)$. A significant increase in contact was also measured in the 12 week group when compared with the 8 week group $(\mathrm{p}=$ 0.006). In the $\mathrm{SiCaP}(+)$ group, significantly increased bone-implant contact was measured in the 24 week group (mean, $83.77 \% \pm 1.09 \%$ ) when compared with both the 8 week $(59.25 \% \pm 4.75 \%)$ and 12 week group (mean, $71.68 \% \pm 3.59 \%)(\mathrm{p}=0.003$ and $\mathrm{p}=0.025$ respectively). No significant difference was found when the 8 and 12-week groups were compared.

\section{Bone Graft Resorption}

Results showed that significantly less implant remained in the $\mathrm{SiCaP}(+)$ group at 12 weeks (mean, $21.58 \% \pm 2.35 \%)$ when compared with graft remaining in the SiCaP group (mean, $30.87 \% \pm 1.49 \%)(\mathrm{p}=$ 0.018). No significant differences were found when the two groups were compared at 8 and 24 weeks. 


\section{Effect of Microporosity on Bone Formation}

Analysis showed a trend in both groups where implant resorption increased over time. In the SiCaP group, significantly less implant remained at 24 weeks (mean, $22.74 \% \pm 1.04 \%$ ) when compared with both the 12 (mean, 30.87\% $\pm 1.49 \%)$ and 8 week groups (mean, $29.98 \% \pm 0.67 \%)(\mathrm{p}=0.006$ and $\mathrm{p}=$ 0.004 respectively). No significant difference was found when the 8 and 12 week data was compared. In the $\mathrm{SiCaP}(+)$ group, significantly less graft material remained at 12 weeks (mean, $21.58 \% \pm 2.35 \%$ ) and 24 weeks (mean, $21.38 \% \pm 2.03 \%)$ when compared with the 8 week group (mean, $29.95 \% \pm 0.98 \%)(p=$ 0.022 and $p=0.004$ respectively). No significant difference was found when the 12 and 24 week data was compared.

\section{Light Microscopy}

Light microscopy confirmed the presence of bone formation throughout both $\mathrm{SiCaP}$ and $\mathrm{SiCaP}(+)$ graft materials and evaluation did not identify any significant differences in the pattern of bone formation associated with either group. Transverse sections showed bony fusion masses on both sides of the spine in most cases. There was some variation in the size of the fusion mass although this did not appear to correlate with the graft type used. At 8 weeks, dense bone formation was generally observed in the graft closest to the vertebrae but by 24 weeks, bone formation was more uniformly spread throughout the fusion mass. In some cases sporadic islands of bone formation were observed within the peripheral regions of the graft bed, suggestive of osteoinductive bone formation in these regions. Both intramembranous and endochondral ossification was observed within the fusion masses of both the $\mathrm{SiCaP}$ and $\mathrm{SiCaP}(+)$ treated spines. Even in the same fusion mass, both types of bone formation could often be seen. The presence of endochondral ossification was unexpected, but was consistently observed throughout both specimen types. Initially bone appeared to preferentially form along the granule concave macropore surfaces with subsequent bone being deposited in a concentric manner (Figure 3). 
In many cases and in both groups, bone was seen bridging between granules and binding the fusion mass together. Extensive bone growth was seen throughout the strut- and macropore network in both graft materials over the 8,12 and 24 week investigative period. Although not quantified in this study, strut pores appeared largely filled with bone with no obvious differences when the $\mathrm{SiCaP}$ and $\mathrm{SiCaP}(+)$ samples were compared. Where minimal bone formation had occurred particularly in the 8-week group, granule degradation was apparent. These regions were often associated with macrophage-like cells containing graft particles and in addition the presence of multinucleate cells on the surface of the graft material could be identified in some regions (Figure 4).

In specimens in both groups and as early as 8 weeks, qualitative examination of the longitudinally cut specimens showed the presence of a fusion bridge from one vertebral body to the adjacent vertebra (Figure 5). In other specimens although new bone formation was evident, fusion had not yet completely bridged the vertebrae together. This was also the case at 12 weeks however at 24 weeks, all animals appeared to have fusion masses that had to some degree, bridged along the length of the spine.

\section{X-Ray Computer Tomography}

In both the $\mathrm{SiCaP}$ and $\mathrm{SiCaP}(+)$ groups, a trend over time was seen where callus formation became more radio-opaque and better defined as a result of consolidation and bony remodelling. In most animals in both groups, the callus was more defined and consolidated at 24 weeks when compared with the 8 and 16 week time points (Figure 6).

Semi-quantitative analysis of bone bridging showed that at 8 weeks post surgery and in both experimental groups, no bone bridging between the two dorsal articular facets was identified. In the SiCaP group, only one animal presented radiological indicators for the presence of a poorly defined 
callus. All other animals in this group were considered to be completely un-fused (mean score, $0.17 \pm 0.17)$. In the $\mathrm{SiCaP}(+)$ group at 8 weeks, a significantly higher semi-quantitative score was seen where callus formation was observed in 5 of the 6 animals and only one animal was un-fused (mean score, $0.83 \pm 0.17)(\mathrm{p}=0.027)$ (Figure 7$)$. At 16 weeks, none of the vertebrae were considered fused and the mean bone bridging score was similar in both groups ( $\mathrm{SiCaP}$ mean, $1.50 \pm$ $0.22 ; \mathrm{SiCaP}(+)$ mean, $1.67 \pm 0.21)$. At 24 weeks post surgery, 2 of the 6 animals in the $\mathrm{SiCaP}$ group had well defined callus that was considered fused (mean score, $2.17 \pm 0.31$ ). Results were similar in the $\mathrm{SiCaP}(+)$ group where the callus in 3 of the 6 animals was seen to have fused (mean score, $2.33 \pm 0.33$ ). No significant differences in scores were identified between the two groups at 16 and 24 weeks. When results were compared over time, a significant increase in score was observed in the $\mathrm{SiCaP}$ group when the 8-week data was compared with both the 16 week $(\mathrm{p}=$ $0.005)$ and 24 week data $(\mathrm{p}=0.003)$. No significant increase in score was measured when the 16 and 24-week data were compared. Similarly, in the $\mathrm{SiCaP}(+)$ group, a significant increase in score was measured when the 8 week data was compared with both the 16 week $(p=0.018)$ and 24 week data $(\mathrm{p}=0.007)$. No significant difference was found when 16 and 24-week data were compared.

No signs of implant loosening, infection or implant migration into the intervertebral disc space were observed in any of the animals investigated. In addition, no evidence of disc degeneration, gross bone resorption, gross resorption or chemical degradation of the graft was reported. None of the animals investigated in this study developed osteophytes, endplate sclerosis, disc mineralisation or a loss in disc height in adjacent vertebrae.

\section{DISCUSSION}

Previous work has shown that increasing strut porosity in silicate-substituted calcium phosphate bone graft materials significantly increased their bone regenerative capacity in ectopic and 
orthotopic models [19-22]. The aim of this study was to investigate whether bone regeneration within a SiCaP scaffold with an average strut porosity of $22.5 \%$ strut porosity could be further enhanced when the strut porosity was increased to $36.0 \%$ in an ovine posterolateral spinal fusion model at 8, 12 and 24 weeks post-operatively. Following surgery, animals showed no signs of implant loosening, infection or implant migration as well as no evidence of disc degeneration or gross bone resorption. None of the animals investigated developed osteophytes, endplate sclerosis, disc mineralisation or a loss in disc height in adjacent vertebrae and so normal healing and maturation of the fusion mass was compared in both groups.

Increased strut porosity is thought to provide an increased surface area for the action of angiogenic and other proteins leading to the formation of blood vessels and promoting bone induction by osteoblasts at early time points $[25,26]$. Studies have also shown that scaffolds with high strut porosity have increased levels of serum proteins, such as vascular endothelial factor, adsorbed onto the surface $[27,28]$. As well as providing an increased surface area for the action of angiogenic and other proteins leading to the formation of blood vessels and promoting bone induction by osteoblasts [25, 26], a study by Campion et al. [21] reported that increased porosity encouraged earlier neovascularisation and increased bone growth in a femoral condyle critical-sized defect model. Chemical composition [7, 20], scaffold macroporosity and pore interconnectivity [29] are also known to influence bone formation within the graft, but all remained constant in this study; the only variation between treatment groups was the strut porosity. Although fusion and bone formation within the graft was seen to increase over time in both of the groups investigated, results from our study showed that similar amounts of bone had formed. Our study showed a significantly higher CT grading score in the $\mathrm{SiCaP}(+)$ group at 8 weeks, indicating an earlier increase in the amount of mature and mineralised bone tissue formed. This may have occurred as a result of the tissue having been there longer and/or being formed by a different and faster mechanism. Results also showed a 
significant reduction in the amount $\mathrm{SiCaP}(+)$ scaffold present at 12 weeks suggesting that an accelerated response to adaptive graft remodelling may have also occurred. Therefore, our results only partially supported our hypothesis as significantly increased volumes of new bone formation were not observed in the $\mathrm{SiCaP}(+)$ treated fusions of healthy animals at any of the study time points of 8,12 and 24 weeks, nor were there any significant differences in the bone apposition rates measured between $5-7,9-11$ and $20-22$ weeks. However, the results of the CT analysis suggest that the bone in the $\mathrm{SiCaP}(+)$ treated fusions was significantly more densely mineralised at 8 weeks, suggesting a greater maturity in the bone tissue within these grafts at this early time-point. These results suggest that the presence of greater levels of strut porosity either promoted early bone formation with a more ordered morphology or accelerated the rate of bone formation at the very early ( $<5$ weeks) time point. In previous studies we have found strut porosity to be critical to the capacity of a synthetic graft to display osteoinductive behaviour $[14,19,20]$. In the current fusion model the grafts were placed in contact with well decorticated bone in relatively young and healthy animals which would have presented less of a challenge to bone formation than either an ectopic model or in the fusion of patients with diseased or degenerated bone stock. Thus to fully understand the role of strut microporosity further work is required to investigate the early response of bone tissue formation, maturation and mineralisation in the presence of silicate-substituted scaffolds with increased strut porosity.

In patients, a lack of fusion between vertebrae may eventually lead to mechanical failure of the internal fixation. In this study, CT results showed complete mineralised fusion in 2/6 animals in the $\mathrm{SiCaP}$ group and 3/6 animals in the $\mathrm{SiCaP}(+)$ group at 24 weeks post surgery. Fusion is indicative of active bone formation, and histological results showed an increase in bone formation over time with an exponential decrease in the quantity of implant present, particularly in the $\mathrm{SiCaP}(+)$ group. In both $\mathrm{SiCaP}$ and $\mathrm{SiCaP}(+)$ specimens and at all time points, extensive bone formation had 
occurred within both the strut- and macropores with remodelled mature bone seen bridging the granules within the graft together. Both of these grafting options produced significant integration of bone within the graft mass with little evidence of fibrous tissue interposition, especially at 24 weeks post surgery. Results showed the $\mathrm{SiCaP}(+)$ scaffold to remodel significantly faster than $\mathrm{SiCaP}$, it is possible that this may further augment the rate of onset of adaptive remodelling. $\mathbf{A}$ post-hoc power analysis was performed and results showed that even with the lowest nonsignificant value $>40$ samples per group are required for a study power of $80 \%$ with a $p<$ 0.05. This confirms that the results for bone integration obtained when the two experimental groups were compared, showed little difference. Biomechanical assessments at the time points investigated would have provided additional information on the progression of healing and the development of functional fusion $[15,30]$. However, the mechanical integrity of the regenerated fusion mass was not measured in this study.

\section{Conclusion}

Increasing the strut porosity did not significantly increase the amount of bone formed however, it did accelerate the rate of early mineralised bone tissue formation, resulting in a significant increase in the amount of mineralised tissue at 8 weeks in the $\mathrm{SiCaP}(+)$ group as measured using our semiquantitative score and based on the radiographic density of bone. Further work is required to investigate the early response of bone tissue formation, maturation and mineralisation in the presence of silicate-substituted scaffolds of increased strut porosity. Our work also raises the question of whether further increases in strut porosity is advantageous for improving osteointegration. Experiments to elucidate this are required however it should be recognized that structural integrity of the graft material may be compromised by high strut porosities. However, for spinal fusion where load is often taken by the instrumentation before bone has formed, bone graft substitute weakness may not be problematic. 


\section{Acknowledgements}

ApaTech Ltd supplied the graft materials and funded this work. The author who works for ApaTech Ltd has received payments or services either directly or indirectly (via ApaTech Ltd) in support of this work. No author has had any other relationships, or has engaged in any other activities that could be perceived to influence what is written in this work. Authors from the John Scales Centre and Queen Mary University were involved in the study design, data collection, analysis and interpretation. All authors were involved in writing the report and in the decision to submit the paper for publication.

\section{Conflicts of Interest}

ApaTech Ltd supplied the graft materials and funded this work. The author who works for ApaTech Ltd has received payments or services either directly or indirectly (via ApaTech Ltd) in support of this work. No author has had any other relationships, or has engaged in any other activities that could be perceived to influence what is written in this work. Authors from the John Scales Centre and Queen Mary University were involved in the study design, data collection, analysis and interpretation. All authors were involved in writing the report and in the decision to submit the paper for publication.

\section{REFERENCES}

1. Delawi D, Kruyt MC, Huipin Y, Vincken KL, de Bruijn JD, Ober FC, Dhert WJ. Comparing autograft, allograft and tricalcium phosphate ceramic in a goat instrumented posterolateral fusion model. Tiss Eng 2013;19(11):1-8.

2. Urist MR. Bone formation by autoinduction. Science 1965;150(698):893-899. 
3. Brown KL, Cruess RL. Bone and cartilage transplantation in orthopaedic surgery. A review. J Bone Joint Surg Am 1982;64(2):270-279.

4. Damien CJ, Parsons JR. Bone graft and bone graft substitutes: a review of current technology and applications. J Appl Biomater 1991;2(3):187-208.

5. Cornell CN, Lane JM. Current understanding of osteoconduction in bone regeneration. Clin Orthop Rel Res 1998;355:S267-273.

6. Lane JM, Tomin E, Bostrom MP. Biosynthetic bone grafting. Clin Orthop Rel Res 1999;367:S107-117.

7. Habibovic P, de Groot K. Osteoinductive biomaterials - properties and relevance in bone repair. J Tissue Eng Regen Med 2007;1:25-32.

8. Nagineni VV, James AR, Alimi M, Hofstetter C, Shin BJ, Njoku I Jr, Tsiouris AJ, Hartl R. Silicate-substituted calcium phosphate ceramic bone graft replacement for spinal fusion procedures. Spine 2012;37(20):E1264-1272.

9. Hinz P, Wolf E, Schwesinger G, Hartelt E, Ekkernkamp A. A new resorbable bone void filler in trauma: early clinical experience and histologic evaluation. Orthopaedics 2002;25(5):S597-S600. 10. Yamamoto T, Onga T, Marui T, Mizuno K. Use of hydroxyapatite to fill cavities after excision of benign bone tumours. Clinical results. J Bone Joint Surg Br 2000;82:1117-1120.

11. Anker CJ, Holdridge SP, Baird B, Cohen H, Damron TA. Ultraporous beta tricalcium phosphate is well incorporated in small cavitary defects. Clin Orthop Relat Res 2005;434:251-257. 12. Patel N, Best SM, Bonfield W, Gibson IR, Hing KA, Damien E, Revell PA. A comparative study on the in vivo behaviour of hydroxyapatite and silicon substituted hydroxyapatite granules. Journal Mater Sci 2002;13:1199-1206.

13. Vallet-Regi M, Arcos D. Silicon substituted hydroxyapatites: a method to upgrade calcium phosphate based implants. J Mater Chem 2005;15:1509-16. 
14. Coathup MJ, Samizadeh S, Fang YS, Buckland T, Hing KA, Blunn GW. The osteoinductivity of silicate-substituted calcium phosphate. J Bone Joint Surg Am 2011;93:2219-26.

15. Wheeler DL, Jenis LG, Kovach ME, Marini J, Turner AS. Efficacy of silicate calcium phosphate graft in posterolateral fusion in sheep. The Spine 2007;7:308-317.

16. Pimenta L, Marchi L, Oliveira L, Coutinho E, Amaral R. A Prospective, Randomized, Controlled Trial Comparing Radiographic and Clinical Outcomes between Stand-Alone Lateral Interbody Lumbar Fusion with either Silicate Calcium Phosphate or rh-BMP2. J Neurol Surg A Cent Eur Neurosurg 2013;74(6):343-50.

17. Barradas AM, Yuan H, van Blitterswijk CA, Habivovic P. Osteinductive biomaterials, experimental models and biological mechanisms. Eur Cells Mater 2011;21:407-13.

18. Bohner M. Silicon-substituted calcium phosphates - a critical view. Biomaterials 2009;30:6403-13.

19. Chan O, Coathup MJ, Nesbitt A, Ho C-Y, et al. 2012. The effects of microporosity on osteoinduction of calcium phosphate bone graft substitute biomaterials. Acta Biomater 8(7):278894.

20. Coathup MJ, Hing KA, Samizadeh S, Chan O, Fang YS, Campion C, Buckland T, Blunn GW. Effect of increased strut porosity of calcium phosphate bone graft substitute materials on osteoinduction. J Biomed Mat Res-A 2012;100(6):1550-5.

21. Campion CR, Chander C, Buckland T, Hing KA. Increasing strut porosity in silicatesubstituted calcium-phosphate bone graft substitutes enhances osteogenesis. J Biomed Mater Res B Appl Biomater 2011;97(2):245-54.

22. Hing KA, Annaz B, Saeed S, Revell PA, Buckland T. Microporosity enhances bioactivity of synthetic bone graft substitutes. J Mater Sci Mater Med 2005;16(5):467-75. 
23. Hing KA, Revell RA, Smith N, Buckland T. Effect of silicon level on rate, quality and progression of bone healing within silicate-substituted porous hydroxyapatite scaffolds. Biomaterials 2006;27(29):5014-5026.

24. Hing KA, Best SM, Bonfield W. Characterization of porous hydroxyapatite. J Mater Sci Mater Med 1999;10(3):135-145.

25. Bignon A, Chouteau J, Chevalier J, Fantozzi G, Carret JP, Chavassieux P, Boivin G, Melin M, Hartmann D. Effect of Micro and Macroporosity of Bone Substitutes on their Mechanical Properties and Cellular Response. J Mater Sci: Mater Med 2003;14(12):1089-1097.

26. Ohtsubo S, Matsuda M, Takekawa M. Angiogenesis after sintered bone implantation in rat parietal bone. Histol Histopathol 2003;18(1):153-163.

27. El-Ghannam A, Ducheyne P, Shapiro IM. Effect of serum proteins on osteoblast adhesion to surface-modified bioactive glass and hydroxyaatite. J Orthop Res 1999;17:340-346.

28. Matsura T, Hosokawa R, Okamoto K, Kimoto I, Akagawa Y. Diverse mechanisms of osteoblast spreading on hydroxyapatite and titanium. Biomater 2000;21:1121-1127.

29. Daculsi G, Laboux O, Malard O, Weiss P. Current state of the art of biphasic calcium phosphate bioceramics. J Mater Sci: Mater Med 2003;14(3):195-200.

30. Voor MJ, Arts JJ, Klein SA, Walschot LH, Verdonschot N, Buma P. Is hydroxyapatite cement an alternative for allograft bone chips in bone grafting procedures? A mechanical and histological study in a rabbit cancellous bone defect model. J Biomed Mater Res B Appl Biomater 2004;71:398-407.

\section{Figure Legends}

Figure 1: A low power photomicrograph of a transverse section through the L4/5 of an animal in the SiCaP group at 24 weeks showing extensive bone formation and new bone in contact with the lamellae. 
Figure 2: Bone apposition rates [A], percentage new bone formation [B], bone scaffold contact $[\mathrm{C}]$ and implant area remaining $[\mathrm{D}]$ within $\mathrm{SiCaP}$ and $\mathrm{SiCaP}(+)$ scaffolds over the 8, 12 and 24-week time periods investigated.

Figure 3: High power photomicrographs showing [A] intramembranous ossification adjacent to a SiCaP surface at 8 weeks, [B] endochondral ossification in an 8 week $\mathrm{SiCaP}(+)$ sample, [C] bone formation onto the concave surfaces of a SiCaP scaffold at 12 weeks and [D] bone bridging between $\mathrm{SiCaP}(+)$ granules at 12 weeks post surgery.

Figure 4: High power photomicrographs showing new bone formation on the surface of the scaffold at 8 weeks post-surgery with bone seen within adjacent struts in [A] the SiCaP group and $[\mathrm{B}]$ the $\mathrm{SiCaP}(+)$ group. Figure 4C shows bone formation within the SiCaP scaffold struts at 12 weeks post surgery and [D] extensive bone growth throughout the micropore structure in a $\mathrm{SiCaP}(+)$ scaffold at 24 weeks post surgery.

Figure 5: A photograph of longitudinal sections A, B and C approximated together showing bone bridging in a $\mathrm{SiCaP}(+)$ specimen at 8 weeks post surgery.

Figure 6: $\mathrm{CT}$ images of an animal in the $\mathrm{SiCaP}(+)$ group at 8, 16 and 24 weeks post-implantation showing fusion at 24 weeks.

Figure 7: A graph showing the results obtained when bone bridging was graded from CT data obtained from animals in the $\mathrm{SiCaP}$ and $\mathrm{SiCaP}(+)$ at 8,16 and 24 weeks post implantation. 